This item was submitted to Loughborough's Research Repository by the author.

Items in Figshare are protected by copyright, with all rights reserved, unless otherwise indicated.

\title{
Mixed mode partition in one dimensional fractures
}

PLEASE CITE THE PUBLISHED VERSION

http://dx.doi.org/10.4028/www.scientific.net/KEM.462-463.616

\section{PUBLISHER}

(c) Trans Tech Publications, Switzerland

VERSION

AM (Accepted Manuscript)

LICENCE

CC BY-NC-ND 4.0

\section{REPOSITORY RECORD}

Wang, Simon, and Christopher M. Harvey. 2011. "Mixed Mode Partition in One Dimensional Fractures". figshare. https://hdl.handle.net/2134/9009. 
This item was submitted to Loughborough's Institutional Repository (https://dspace.lboro.ac.uk/) by the author and is made available under the following Creative Commons Licence conditions.

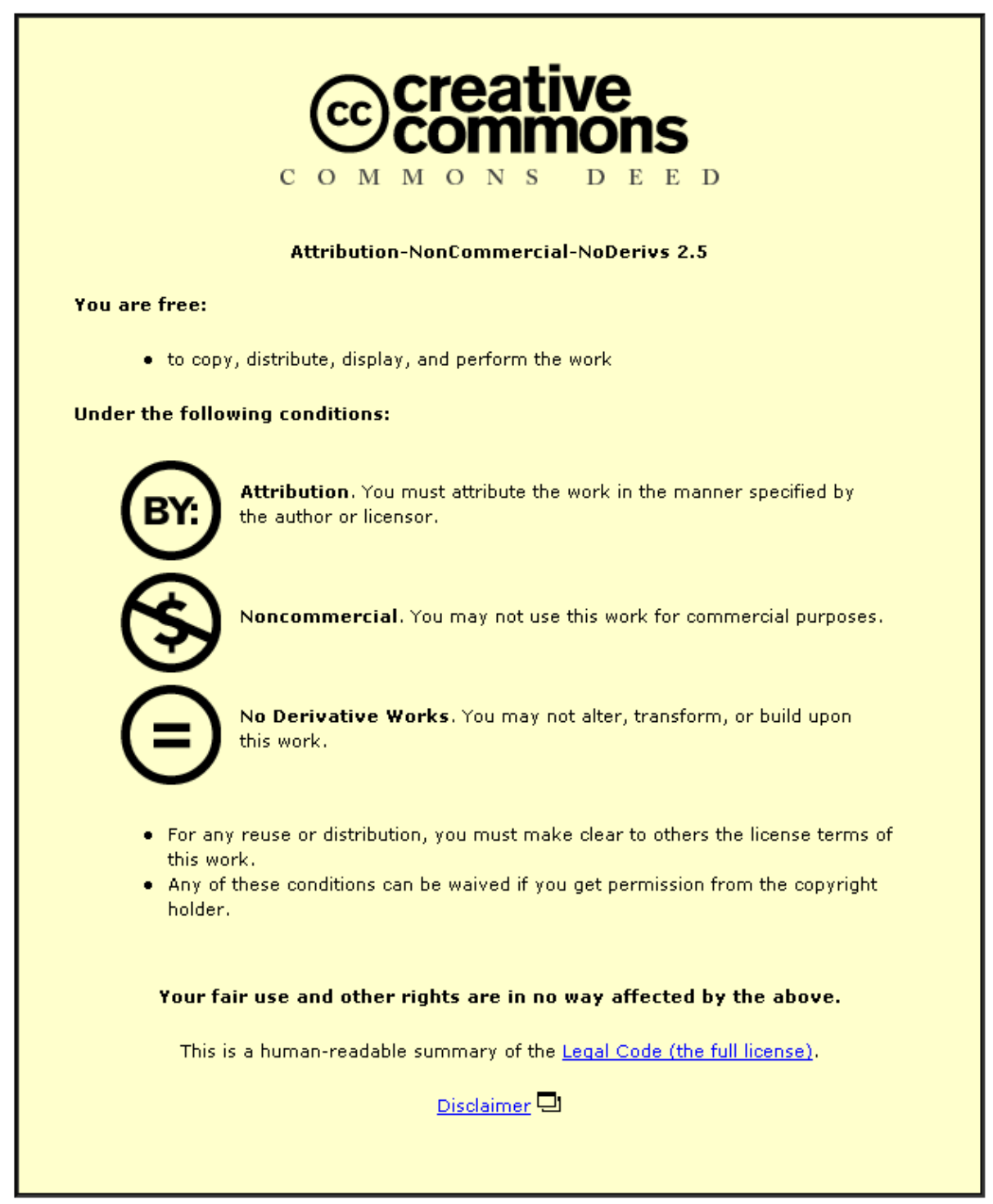

For the full text of this licence, please go to: http://creativecommons.org/licenses/by-nc-nd/2.5/ 


\title{
Mixed Mode Partition in One Dimensional Fractures
}

\author{
Simon Wang and Chris Harvey \\ Department of Aeronautical and Automotive Engineering, \\ University of Loughborough, Loughborough, LE11, 3TU, UK. \\ E-mail: s.wang@lboro.ac.uk; c.m.harvey@lboro.ac.uk
}

Keywords: orthogonal pure mode, pure mode interaction, mixed mode partition

\begin{abstract}
Taking a double cantilever beam (DCB) as a representative of one dimensional fracture, a unique pair of pure fracture modes I and II are successfully found in the absence of axial forces, which are orthogonal to each other with respect to the coefficient matrix of the energy release rate. Although the pair are pure modes there still exist interactions between them. The interactions result in energy flow between the two modes and are successfully determined. With the presence of axial forces, there are two independent pure modes I and two independent pure modes II, which are orthogonal to each other as well. They are found and used to partition the total energy release rate.
\end{abstract}

\section{Introduction}

DCBs are typical representatives of one dimensional fracture problems and often used to determine critical strain energy release rates of materials. Fracture mode partitions play a key role in the development of crack propagation criteria. The Williams partition rules are given in his excellent pioneering work [1]. Subsequently, many other researchers, in particular Hutchinson and Suo have reported the limitations of Williams rules in their excellent work [2]. As far as the authors' knowledge is concerned, the previous works have not reported such simple but not simpler rules as that in the present work. It should be noted that a brief version of the present work has been reported in two international conferences by the authors $[3,4]$.

\section{Theory}

A Brief Introduction to Fracture Mode Partition of A DCB. A DCB with a crack length $a$ is shown in Fig. 1 (a). Contact stresses as shown in Fig. 1 (b) usually occur under general tip loads. However, it is assumed that no contact happens in the present study. The strain energy release rate $G$ at crack tip point B is given by

$$
G=\left[M_{1 B}^{2} / I_{1}+M_{2 B}^{2} / I_{2}-M_{B}^{2} / I\right] /(2 b E) .
$$

Here, $b$ is the width of the beam and the other quantities have their conventional meanings. It is seen that $G$ is a quantity of local nature depending on the quantities on the crack tip cross-section, i.e., $M_{1 B}, M_{2 B}$ and $M_{B}$. Also, $G$ is a quadratic form of positive definite and can be expressed in orthogonal forms. To do so, $M_{1 B}$ and $M_{2 B}$ are expressed as

$$
\left\{\begin{array}{l}
M_{1 B} \\
M_{2 B}
\end{array}\right\}=\alpha_{I}\left\{\begin{array}{c}
1 \\
\lambda_{I}
\end{array}\right\}+\alpha_{I I}\left\{\begin{array}{c}
1 \\
\lambda_{I I}
\end{array}\right\}=\alpha_{I} \phi_{I}+\alpha_{I I} \phi_{I I} .
$$




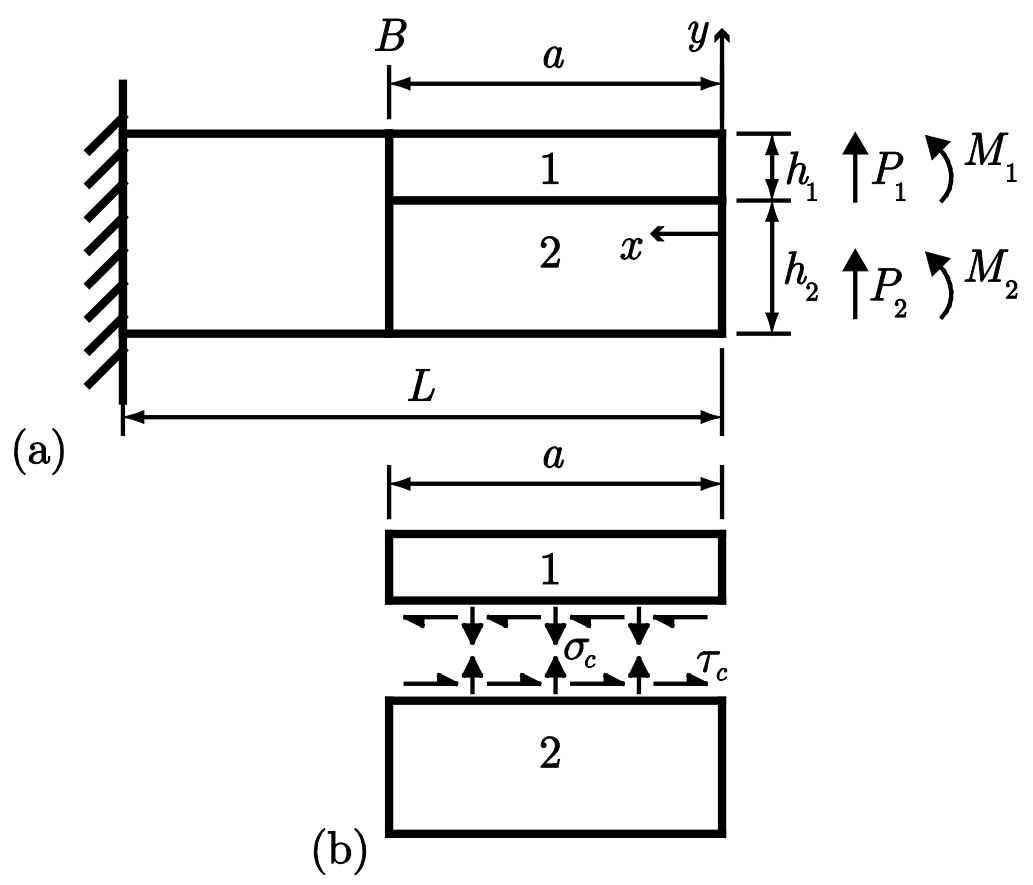

Figure 1: A DCB and its loading conditions. (a) General description, (b) Contact stresses.

When either $\lambda_{I}$ or $\lambda_{I I}$ is given, the other can be found using the orthogonal condition with respect to the coefficient matrix of the form. Therefore, a pair of orthogonal modes, i.e. $\phi_{I}$ and $\phi_{I I}$ can be found. $\alpha_{I}$ and $\alpha_{I I}$ are the mode partition coefficients and can be determined from Eq.2 for any given $M_{1 B}$ and $M_{2 B}$. Then, substituting Eq. 2 into Eq. 1 yields,

$$
G=\left[\alpha_{I}^{2}\left(1 / I_{1}+\lambda_{I}^{2} / I_{2}-\left(1+\lambda_{I}\right)^{2} / I\right)+\alpha_{I I}^{2}\left(1 / I_{1}+\lambda_{I I}^{2} / I_{2}-\left(1+\lambda_{I I}\right)^{2} / I\right)\right] /(2 b E)=\alpha_{I}^{2} G_{\phi_{I}}+\alpha_{I I}^{2} G_{\phi_{I I}} .
$$

where $G_{\phi_{I}}$ and $G_{\phi_{I I}}$ are the energy release rates corresponding to the orthogonal modes $\phi_{I}$ and $\phi_{I I}$, respectively. In general, they are not the respective pure opening mode I and shearing mode II as there exist an infinite number of orthogonal modes. Consequently, it is very natural to enquire whether a unique pair of orthogonal pure modes I and II exist in physical reality and how to find them from the infinite number of orthogonal pairs when they exist. A further enquiry is that when the pair are found, does the Eq. 3 represents the true energy release rate partition?

Two Pairs of Averagely Pure Fracture Modes. To begin with, it is assumed that the mechanical influence of the crack extends to a point A, $\Delta a$ distance ahead of the crack tip B as shown in Fig. 2 (a). It is noted that Fig. 2 (b) only shows the sign convention of the interface stresses instead of any representative distribution of them. $F_{s}$ and $F_{n}$ are the resultant shear and normal forces on the interface of $\Delta a . \bar{s}$ is the distance between a chosen point $\mathrm{p}$ and the point $\mathrm{A}$, and $m_{p}(\bar{s})$ is the resultant moment about the point $\mathrm{p}$ of the normal stress. The two resultant forces $F_{s}$ and $F_{n}$ can be determined by considering the curvature continuity at point A. An averagely pure mode I is defined as satisfying $F_{s}=0$. This leads to the first pair of averagely pure modes. That is

$$
\hat{\phi}_{I}=\{1,-1\}^{T}, \hat{\phi}_{I I}=\left\{1, \gamma^{3}\right\}^{T} \text {. }
$$


where $\gamma=h_{2} / h_{1}$. In a similar thought, an averagely pure mode II is defined as satisfying $F_{n}=0$. This leads to the second pair of averagely pure modes. That is

$$
\phi_{I}=\{1, \theta\}^{T}, \phi_{I I}=\{1, \beta\}^{T} \text {. }
$$

in which $\theta=-\gamma^{2}$ and $\beta=\gamma^{2}(3+\gamma) /(1+3 \gamma)$. Now, two pairs of averagely pure modes are found. It is noted that the first pair are the Williams pair [1].

(a)

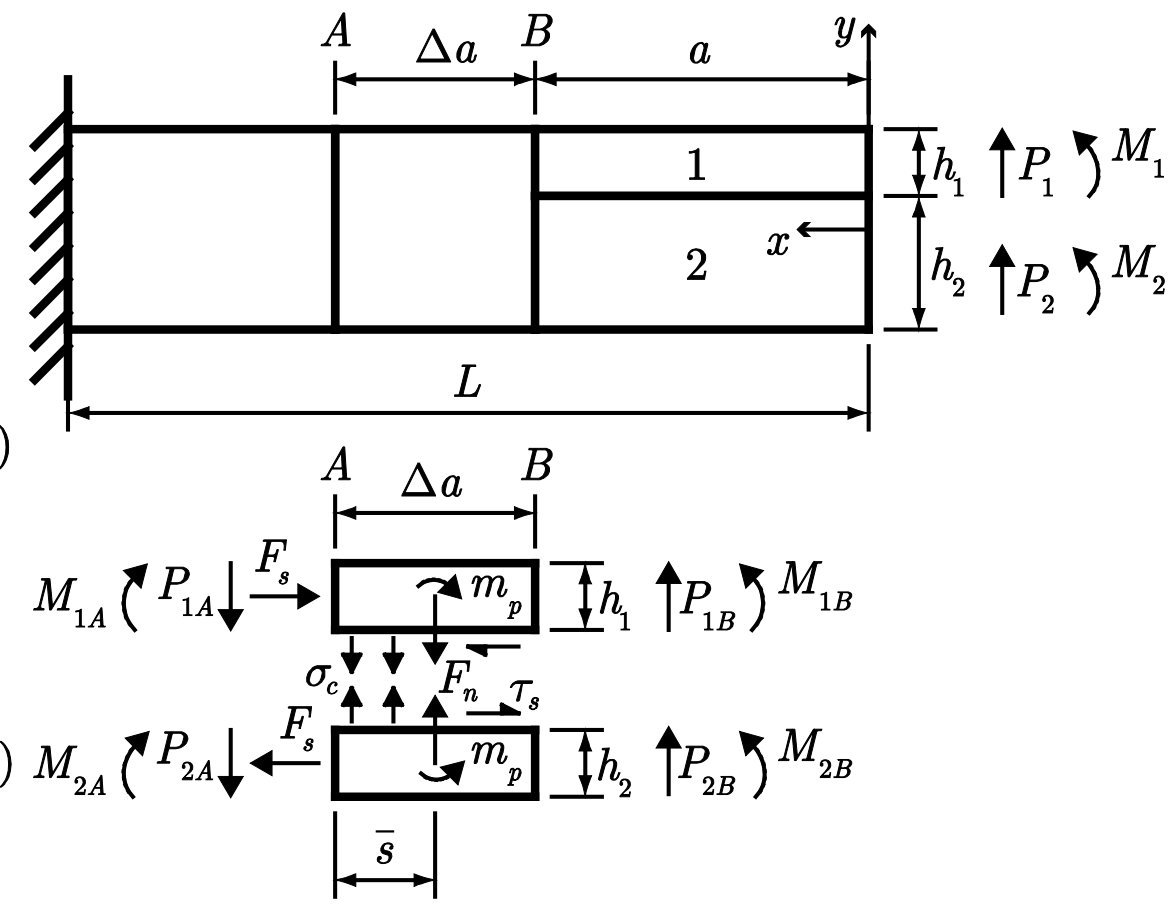

Figure 2: A DCB with $\Delta a$ region. (a) General description, (b) Details of the $\Delta a$ region.

The Unique Pure Pair of Fracture Modes. Now, it proceeds to determine the pure pair modes by deformation analysis. The zero relative shearing displacement just behind the crack tip is,

$$
\bar{u}_{1 d a}-\bar{u}_{2 d a}=\left(u_{1 B}+h_{1} / 2 V_{1 d a}^{(1)}\right)-\left(u_{2 B}-h_{2} / 2 V_{2 d a}^{(1)}\right) .
$$

Eq. 6 gives the mode I of the second pair. It can be concluded now that the second pair are the unique pure pair. Before proceeding to the next section, a brief summary on the second pair is worthy of attention. In pure mode I, the relative shearing displacement just behind the crack tip is zero. However, both normal and shear stresses are in general not zero at the crack tip. In pure mode II, the normal stress is uniformly zero on the interfaces of the two beams within the $\Delta a$ region including the very crack tip. However, the relative opening displacement just behind the crack tip is in general not zero. These characteristics lead to interactions between the two pure modes.

Interactions between the Two Pure Modes. At this point, it is natural to ask whether applying the two pure modes into Eq. 3 gives the correct partition for a given mixed mode. Unfortunately, the answer is negative. The cause is the interactions between these two pure modes which have been determined after a thorough study. Consequently, a mixed mode is partitioned as 
$G_{I}=\alpha_{I M}^{2} G_{\phi_{I M}}+\alpha_{I M} \alpha_{I I M} G_{I \phi_{I M} \phi_{I M}}$.

(7)

$G_{I I M}=\alpha_{I I M}^{2} G_{\phi_{I M}}+\alpha_{I M} \alpha_{I I M} G_{I I \phi_{I M} \phi_{I M}}$.

(8)

The two second terms are the interaction terms of same magnitude with opposite sign.

Addition of Axial Forces. When axial forces are considered, two independent mode I modes are found to be

$$
\phi_{I 1}=\{1, \theta, 0\}^{T}, \phi_{I 2}=\{1,0, \varsigma\}^{T} .
$$

(9)

where $\varsigma=-6 / h_{1}$. Two independent mode II modes are found to be

$$
\phi_{I I 1}=\{1, \beta, 0\}^{T}, \phi_{I I 2}=\{1,0, \xi\}^{T} \text {, when } h_{1}=h_{2}, \phi_{I I 2}=\{0,0,1\}^{T}
$$

where $\xi=2(\gamma+3) /\left[h_{1}(\gamma-1)\right]$. Note that the two independent modes I are orthogonal to the two independent modes II with respect to the coefficient matrix of the strain energy release rate. The total strain energy release rate is then partitioned as

$$
G_{I}=\alpha_{I 1}^{2} G_{\phi_{I 1}}+\alpha_{I 1} \alpha_{I I 1} G_{I \phi_{11} \phi_{I 1}}+\alpha_{I 1} \alpha_{I I 2} G_{I \phi_{I 1} \phi_{I I}}
$$

$$
G_{I I}=\alpha_{I I 1}^{2} G_{\phi_{I I}}+\alpha_{I I 2}^{2} G_{\phi_{I I 2}}+\alpha_{I 1} \alpha_{I I 1} G_{I I \phi_{11} \phi_{I I 1}}+\alpha_{I 1} \alpha_{I I 2} G_{I I \phi_{1} \phi_{I I 2}}+2 \alpha_{I I 1} \alpha_{I I 2} G_{\phi_{I I} \phi_{I I 2}}
$$

Application to More General One Dimensional Fractures. Here, two more general one dimensional fractures are considered as shown in Figs. 3 and 4. A clamped beam with a central delamination is shown in Fig. 3 and a clamped circular plate with a central delamination is shown in Fig. 4. By using the theory in the previous section, the pure mode conditions are found to be

$$
P_{2}=\theta P_{1}, P_{2}=\beta P_{1} \text {. }
$$

for pure modes I and II, respectively. The analytical strain energy release rates for the beam in mode I and mode II are given by

$$
G_{I}=\frac{3 a^{2} \gamma P_{1}^{2}}{b^{2} E h_{1}^{3}(1+\gamma)}, G_{I I}=\frac{9 a^{2} \gamma(1+\gamma) P_{1}^{2}}{b^{2} E h_{1}^{3}(1+3 \gamma)^{2}} .
$$

The analytical strain energy release rates for the plate in mode I and mode II are given by

$$
G_{I}=\frac{3 \gamma\left(1-v^{2}\right) P_{1}^{2}}{2 E h_{1}^{3} \pi^{2}(1+\gamma)}, G_{I I}=\frac{9 \gamma\left(1-v^{2}\right)(1+\gamma) P_{1}^{2}}{2 E h_{1}^{3} \pi^{2}(1+3 \gamma)^{2}} .
$$




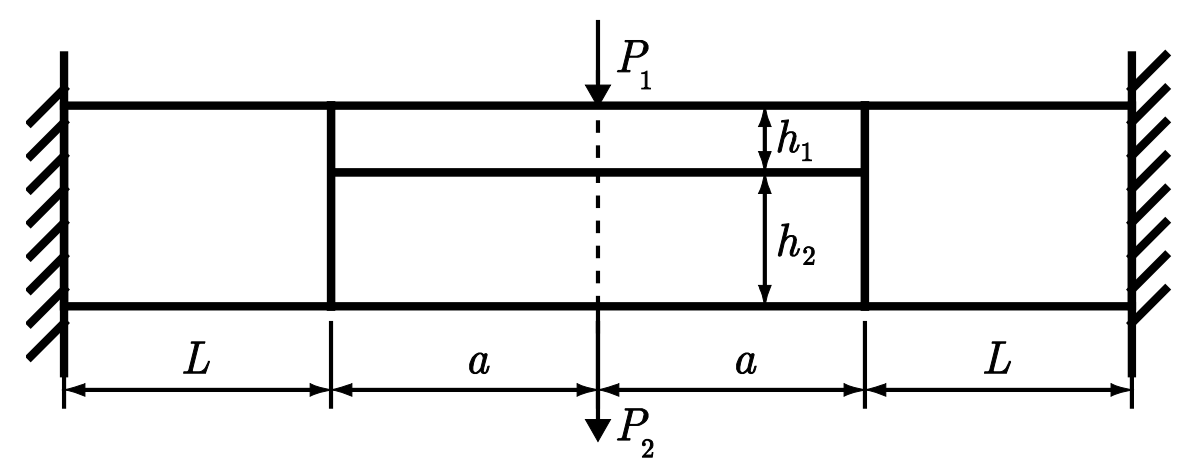

Figure 3: A clamped beam with a central delamination.

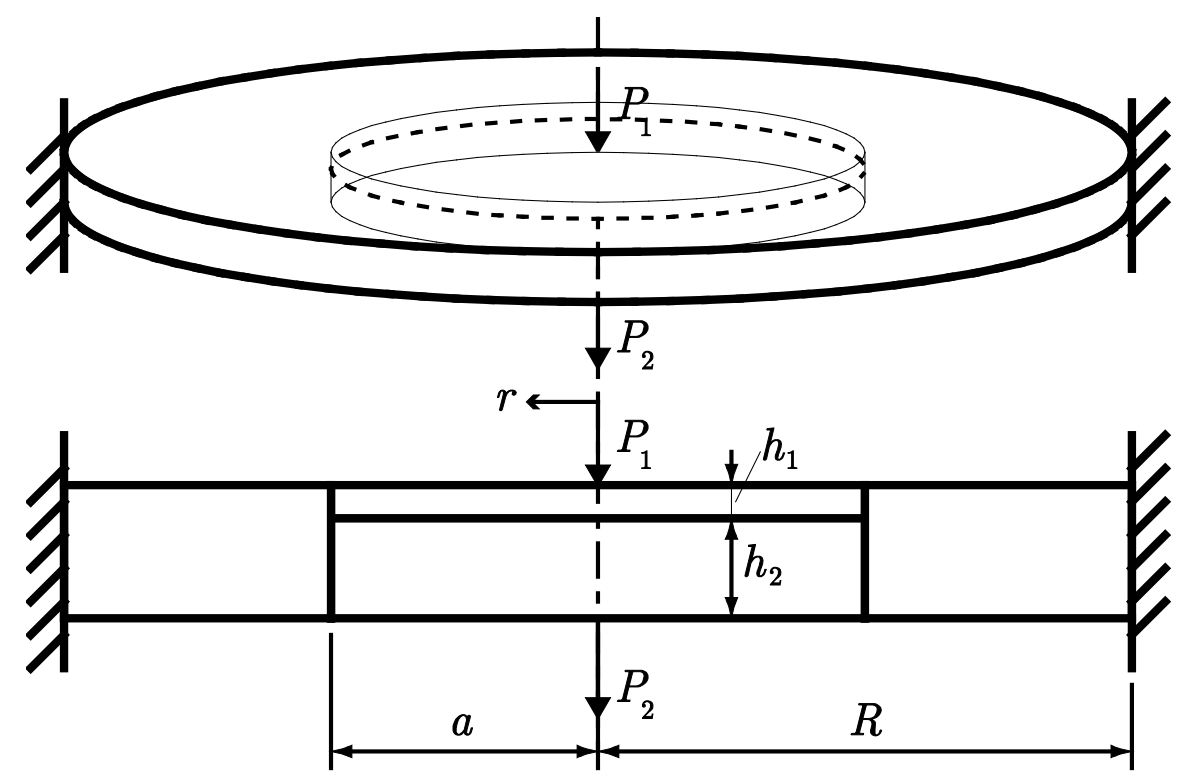

Figure 4: An axisymmetric plate with a central delamination.

\section{Tests}

An FEM simulation capability has been developed using thin composite laminated beam element in conjunction with imaginary normal and shear spring method and crack closure technique $[5,6]$ to numerically determine the energy release rate and its partitions. The first test aims to compare the present theory with Hutchinson and Suo's work [2]. The upper arm of a DCB is loaded with a bending moment $\mathrm{M}=1 \mathrm{Nm}$. The data of the DCB are the Young's modulus $\mathrm{E}=124 \mathrm{GPa}$, the length $\mathrm{L}=100 \mathrm{~mm}$, the width $\mathrm{b}=5 \mathrm{~mm}$, the crack length $\mathrm{a}=40 \mathrm{~mm}$ and the total thickness $h_{1}+h_{2}=0.75 \mathrm{~mm}$. The results, i.e. $G_{I} / G$ are presented in Table 1 . It is seen that the results from the present rules are almost identical to the FEM predictions.

Table 1: Mode partitions for an asymmetric DCB.

\begin{tabular}{|l|l|l|l|l|l|}
\hline$\gamma=h_{2} / h_{1}$ & 4 & 1.5 & 1 & 0.667 & 0.25 \\
\hline Present rule & 0.671 & 0.627 & 0.571 & 0.515 & 0.432 \\
\hline FEM & 0.673 & 0.627 & 0.571 & 0.515 & 0.432 \\
\hline$[2]$ & 0.611 & 0.588 & 0.571 & 0.549 & 0.481 \\
\hline
\end{tabular}

The second test concernes about the more general one dimensional fracture shown in Fig. 4 . Table 2 shows the data of the plate. 
Table 2 Data of the clamped axisymmetric plate.

\begin{tabular}{|l|l|l|l|l|l|l|l|l|}
\hline$E$ & $v$ & $R$ & $a$ & $h_{1}$ & $h_{2}$ & $\gamma$ & $\beta$ & $\theta$ \\
\hline 140GPa & 0.3 & $100 \mathrm{~mm}$ & $20 \mathrm{~mm}$ & $1 \mathrm{~mm}$ & $2 \mathrm{~mm}$ & 2 & $20 / 7$ & -4 \\
\hline
\end{tabular}

The load $P_{1}$ was set to $1000 \mathrm{~N}$. The strain energy release rate results are given in Table 3 .

Table 3 Numerical release rates partition for $\phi_{I}, \phi_{I I}\left(J / \mathrm{m}^{2}\right)$.

\begin{tabular}{|l|l|l|l|l|l|l|}
\hline \multicolumn{3}{|l|}{ Present rule } & \multicolumn{3}{l|}{ FE results with 200 elements } \\
\hline & $G_{I}$ & $G_{I I}$ & $G$ & $G_{I}$ & $G_{I I}$ & $G$ \\
\hline$\phi_{I}$ & 658.6 & 0 & 658.6 & 687.2 & 9.685 & 696.9 \\
\hline$\phi_{I I}$ & 0 & 362.9 & 362.9 & 4.913 & 371.7 & 376.7 \\
\hline
\end{tabular}

A good agreement is observed between the analytical and numerical results.

\section{Conclusions}

The pure modes and mixed mode partition in a DCB have caused considerable confusions due to their in-depth subtleness among researchers in the community of fracture mechanics and were thought to be non-solvable within the context of beam theory. They are now solved in this paper exactly using beam theory. It turns out that the solution is so simple and classic that it is recommended to be taken into fracture mechanics text books for educational purpose. In the absence of axial forces, the unique pair of pure fracture modes are successfully found which are orthogonal to each other with respect to the coefficient matrix of the energy release rate. Although the pure pair are pure, there still exist interactions between them. With the presence of axial forces, there are two independent pure mode I and two independent mode II which are orthogonal to each other as well. They are found and used to partition the total energy release rate. Also, the interactions between them are determined. The simple partition rule gives accurate mode partitions for mixed modes. Numerical experiments validate the theory.

\section{References}

[1] J. G. Williams: International Journal of Fracture Mechanics 36 (1988), p. 101.

[2] J. W. Hutchinson and Z. Suo: Advances in Applied Mechanics, 29 (1992), p. 63

[3] S. Wang and C. Harvey: Fracture mode partition rules for DCB , $17^{\text {th }}$ Interactional Conference on Composite/Nano Engineering, Honolulu, Hawaii,USA, July 2009.

[4] C. Harvey and S. Wang: Modelling of delamination propagation in composite laminated beam structures, Proceedings of the $7^{\text {th }}$ International Conference of Computational Methods in Science and Engineering (ICCMSE 2009), Simos T E (ed), American Institute of Physics, Rhodes, Greece.

[5] Y. Zhang and S. Wang: Composite Structures 88 (2009), p. 121 (Also, a plenary speech in the $16^{\text {th }}$ International Conference on Composite/Nano Engineering, Kunming China, July 2008).

[6] S. Wang and Y. Zhang: Composite Structures 88 (2009), p. 131 (Also, a plenary speech in the $16^{\text {th }}$ International Conference on Composite/Nano Engineering, Kunming China, July 2008). 